\title{
PREDICTION OF BRAKE ONSET TIMES FOR REAR END COLLISIONS
}

\author{
Nicholas J. Kelling and Gregory M. Corso \\ Georgia Institute of Technology \\ Atlanta, GA \\ E-mail: nk28@mail.gatech.edu
}

\begin{abstract}
Summary: Driving has become an integral part of our daily lives, and so too have the dangers associated with driving. Understanding driver behavior could lead to system modifications to alleviate some of these inherent dangers. Specifically, prediction of driver braking behavior might be used to improve automatic braking systems and adaptive cruise control systems. The research presented in this paper details the development of an algorithm to predict the brake onset times in situations where rear-end collisions might occur. The algorithm is adaptive to an individual and not set to generic values. This algorithm was generated using data from a previous study (Kelling, 2006). Displayed stimuli consisted of different situations for a lead vehicle (stopped, slower moving, and reversing lead vehicle), multiple rates of closure $(32.2,64.4$, and $96.6 \mathrm{kph})$, and two luminance conditions (day or night driving). Brake onset times were recorded. A self-modifiable algorithm was developed and was found to have an R-squared value of .625. The degree of goodness-of-fit for this algorithm is worthy of note because it also considers differences in the driving environment. The individualized adaptive ability of the algorithm provides a greater overall fit for predicting braking behavior, and it may be more useful in automated systems than existing algorithms.
\end{abstract}

\section{INTRODUCTION}

Rear-end collisions accounted for more than $43 \%$ of the nearly 4.2 million reported multiple-car accidents in the United States (Traffic Safety Facts, 2007). Rear-end collisions could be caused by environmental factors, such as weather or road ice, or situational factors, such as driver distraction caused by alcohol or cell phone use while driving. The operator's ability to drive can also be a factor in such events. Young, inexperienced drivers may have not yet learned the correct responses for certain situations. Older adults may have an increased reaction time to changing events while driving. The driver's perceptual abilities are also vital to the success of driving. Failure to correctly assess the situation could have catastrophic results. Because perceptual failures exist at the primary level of the system, malfunctions at this level can cascade throughout the rest of the system. Identifying information that could be misrepresented or incorrectly perceived might aid in comprehending not only deviations and aberrations in driving, but also what constitutes normal driving. This notion of normalization in driving has led to the creation of predictive models of driver behavior.

\section{Lead Vehicle Perception}

Kelling (2006) provided evidence on the importance of the lead vehicle movement condition on a driver's decision on when to initiate braking. When braking is initiated depends on what the 
lead vehicle is doing. This finding has an interesting impact on the direct use of the perceptual cue tau. When using Regan and Gray's (2000) equation for tau, no mention is made about the lead vehicle behavior. Interpupillary distance, lead vehicle distance, and rate of change of relative disparity can be equal across lead vehicle conditions for a specific rate of closure. A similar argument could be generated for Bootsma's (1991) interpretation of tau. Thus, tau times across these scenarios would be equivalent, but because of the differences in what the lead vehicle is doing the perception may be different, causing changes in cognitive processing and the initiation of the resulting movement. Creating a predication based tau, could possibly ignore the variability due to the scenario based on the lead vehicle's movement condition.

The investigation of direct reaction time has been extensive over the years (see Green, 2000). Even so, there are large limitations in using canonical brake reaction times. Large variability and adaptability in the driving environment are problematic when all scenarios are collapsed into a single reaction type. Taking the opposing stance formulates its own problems. Attempting to catalogue the reaction times for every scenario a driver might encounter is quite a daunting, if not an impossible, task. Nevertheless, a predictive method cannot overlook the general reaction time of the driver whose behavior it is attempting to predict. Decreases of reaction time due to age or other conditions might have generalizable consequences to reacting to crash-related scenarios. A predictive algorithm must then be able to integrate these general factors, as well as those of the perceptual qualities that are relevant. Because of the possible age or conditional effects on an individual, it might be said that such an algorithm must learn and adapt as the users change.

\section{Current Models}

The previous argument highlights the dilemmas associated with using a canonical brake reaction time to predict human braking behavior. The next evolutionary step involves the development of algorithm models. These models allow more flexibility than generic reaction times, but they present a new issue regarding what perceptual information may provide predictive power. Two simple models include constant distance and constant tau. Constant distance minimizes the necessary perceptual information to distance by only requiring a distance variable. While this method does supply some formulation of individual differences, an individual's variability is limited to a single constant. Constant tau also incorporates a general preference of the driver while using a more complex perceptual cue. Once again, the variability of the individual's brake onset pattern is limited to a single interaction between a preference of time to contact, but does incorporate the velocity of the lead vehicle relative to the driver. Although, these models provide a more detailed account of a driver's braking behavior than a canonical reaction time, Kelling (2006) indicated less than optimal results using these models.

\section{METHODS}

\section{Participants}

Fifty Georgia Tech undergraduate students of both genders participated in this experiment. Only participants with normal or corrected to normal vision who had at least two years of driving experience were recruited. No additional driving experience measures were taken. All 
participants were treating according to Institute Review Board procedures.

\section{Apparatus}

Participants were tested using an individualized testing station consisting of a PC computer with a 43.2-cm (17-in) CRT monitor. Multiple stations were simultaneously used. The computer was connected to an attached custom built functional brake pedal. The sequence and presentations of the animations were controlled using a program written in Inquisit (Millisecond ${ }^{\mathrm{TM}}$, 2005). Each

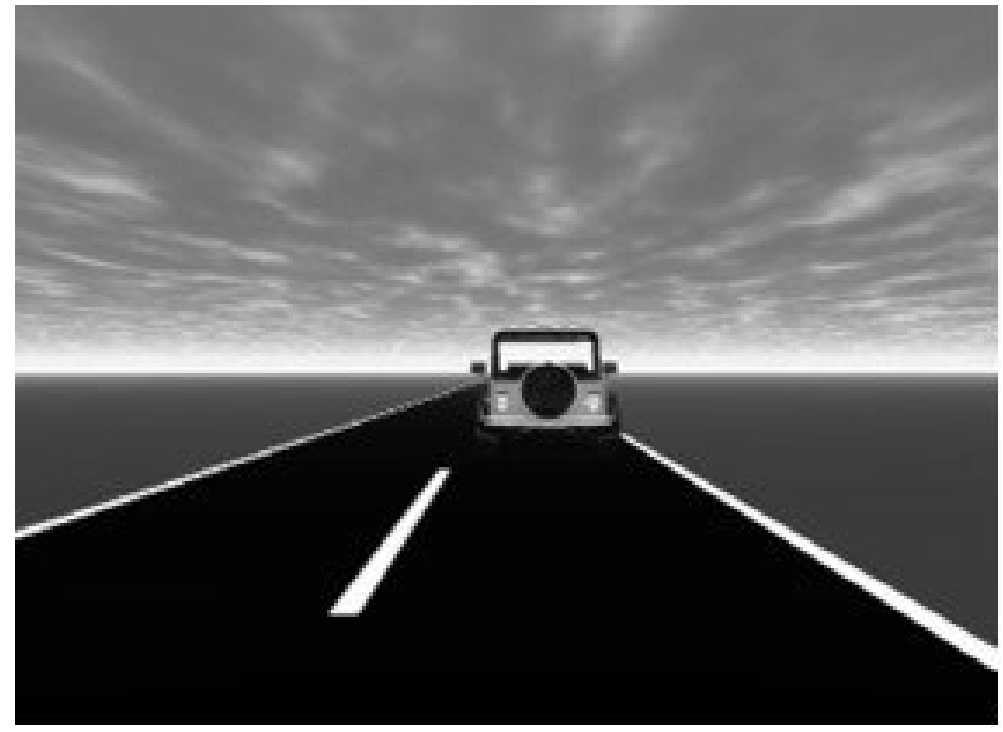

Figure 1. Animation Sample

trial consisted of an animation depicting a road converging in the distance. Carrera ${ }^{\mathrm{TM}} 4$ (Eovia $^{\mathrm{TM}}$, 2005). At the beginning of each animation, the self-motion appeared as if the participant was inside and driving a vehicle. In the distance a vehicle appeared, the lead vehicle. The appearance of the vehicle was sufficiently distant so the participant was not surprised by its occurrence.

Figure 1 depicts an example of an animation. A total of 30 animations were used, including catch trials consisting of no collisions, and each was a maximum of 10 seconds in duration.

\section{Procedure}

After granting consent, the participant was placed at the testing apparatus. Each participant was asked to depress the brake pedal when they would normally brake given the circumstances presented in the animation. The participant was informed that the goal was to depress the brake pedal as they normally would while driving. When the participant depressed the pedal, the time was recorded and the next trial began. The variables manipulated were rate of closure, lead vehicle motion, and luminance. Rate of closure was defined as the difference between the speeds of the lead vehicle relative to the participant's vehicle. If the participant's vehicle was traveling at $32.2 \mathrm{kph}(20 \mathrm{mph})$ and the lead vehicle was stopped, this results in a 32.2-kph (20-mph) rate of closure. The same rate of closure occurred when the participant's vehicle was traveling at 40 $\mathrm{mph}$ and the lead vehicle moved at $20 \mathrm{mph}$. Rates of closure assumed values of 32.2, 64.4, 96.6 $\mathrm{kph}(20,40$ and $60 \mathrm{mph})$, with the participant's vehicle assuming speeds of $0,32.2,64.4,96.6$, or 
$128.8 \mathrm{kph}(0,20,40,60$, or $80 \mathrm{mph})$. Three types of lead vehicle motion condition were employed: a) driving toward a stopped lead vehicle, b) driving toward a slower moving lead vehicle, or c) stopped while a lead vehicle was reversing. Finally, luminance was limited to day or night environments. Lead vehicle brake lights and participant's vehicle headlights remained constant for either luminance condition. Additionally, catch trials, in which the lead vehicle changed speeds to match the participant's vehicle speed for each rate of closure by luminance combination, were added to prevent an anticipatory response. The combination of rate of closure by lead vehicle condition by luminance level was replicated 15 times for a total of 270 trials plus 90 catch trials for a total of 360 trials. Upon completion of the experiment, the participant was debriefed and any questions were answered.

\section{Analysis and Results}

Brake onset times for each participant were transformed to tau times. Previous analysis of the data (Kelling, 2006) determined that luminance was a non-significant factor. It was therefore not included in the prediction algorithm. A repeated measures regression was used to create weights for the variables of rate of closure, lead vehicle motion condition, and an additional factor consisting of a base measure of an individual's braking response. This base measure was calculated by averaged brake onset times across vehicle motion condition but within a single rate of closure. Three inputs are included in the algorithm. The rate of closure is based from the driver's frame of reference in terms of miles per hour. The second variable, the lead vehicle motion condition, takes one of three constants (parked lead, slower moving lead, or reversing lead vehicle). The final input is the base measure, averaged tau time within rate of closure, in milliseconds. The resulting value is the tau time of normal brake onset in milliseconds. The Rsquared correlation for the model found in this experiment was .625.

\section{DISCUSSION}

The basis of the algorithm can be seen in equation 1. This equation predicts when a driver would normal begin to depress a brake based on the information presented earlier.

$$
\begin{aligned}
& P B O T=\mathrm{c}_{1}-\mathrm{c}_{2}(\mathrm{RoC})-\mathrm{c}_{3}(\mathrm{VMC})-\mathrm{c}_{4}(\mathrm{BM}) \\
& \text { where } \\
& \text { RoC }- \text { Rate of Closure } \\
& \text { VMC }- \text { Vehicle Motion Condition } \\
& \mathrm{BM}-\text { Base Measure }
\end{aligned}
$$

The constants have been withheld from this discussion, but serve as weights for the algorithm. The output is predicted brake onset time, or PBOT. By equipping an automotive braking system with this type of algorithm, the system would have the ability to predict when a driver would normally react. If the driver did not react during this window, the system would have the time to react in a multitude of fashions, whether by warnings or brake action.

General results from this study aid in a greater understanding of the driving environment we experience daily. The fit of the algorithm is quite substantial considering how dynamic the driving environment can be. Even within the relative confine of rear-end collisions, the fit of this 
model could greatly aid driver safety. Over $62 \%$ of the variance in the braking behavior observed was accounted for by three variable types. When compared to existing models such as constant distance or constant tau, less significant results are found using the same data set: .605 and .412 (Kelling, 2006). This finding speaks volumes on how our understanding of dynamic braking behavior could be reigned in. If such an objective could be reached, predictive models could become more effective. By effectively predicting brake onset behavior, assistive technologies such as adaptive cruise control and automatic braking systems will be better able to react to scenarios where collisions may occur. Systems that are based on more optimal information are likely to be more successful. Success in such an instance could mean the life of drivers, as well as that of those around them. To assume this goal is dependent on a predictive system that averages types of individual drivers to provide an umbrella prediction would not be the most advantageous method. Instead of treating the issue with a global solution, a solution that can adapt to every individual driver provides a much more robust result to driver assistive systems, while still covering the entire population. Adapting to the driver's behavior provides these systems with a glimpse of what the driver may do or should do, and by altering the technology's behavior a greater level of trust and use of these technologies may occur. The high correlation derived from this model also suggests using the algorithm for accident reconstruction. Brake onset times could be generated for drivers and compared to the driver's actual behavior.

\section{REFERENCES}

Bootsma, R.J. (1991). Grasping Tau. Journal of Experimental Psychology, 17(2), 315-322.

Carrara 4 [Computer software]. (2005). Mountain View, CA: Eovia Corporation.

Green, M. (2000). "How long does it take to stop?” Methodological analysis of driver perception-brake times. Transportation Human Factors, 2(3), 195-216.

Inquisit 2.0.51002.1 [Computer software]. (2005). Seattle, WA: Millisecond Software LLC.

Kelling, N.J. (2006). The Influence of Visual Perception on Vehicle Rates of Closure. Unpublished masters' thesis, Georgia Institute of Technology.

Regan, D., \& Gray, R. (2000). Visually guided collision avoidance and collision achievement. Trends in Cognitive Science, 4(3), 99-107.

Traffic Safety Facts 2005. (2007). Retrieved from http://www.nhtsa.dot.gov/portal/nhtsa static_file_downloader.jsp?file=/staticfiles/DOT/NHTSA/NCSA/Content/TSF/2006/TSF200 5.pdf 